BRIEF COMMUNICATION

\title{
Current and future in FHR monitoring
}

\author{
Takashi Okai \\ Department of Obstetrics \& Gynecology \\ Showa University School of Medicine
}

Electronic fetal heart rate monitoring during labor was developed in early 1960 's. In 1968, the first clinical electronic monitor became available, and Paul and Hon ${ }^{1}$ reported its beneficial effect on perinatal outcomes in 1974.

It has been considered that electronic monitoring helps obstetricians identify and interpret changes in FHR patterns that may be associated with fetal conditions as hypoxia, acidosis, umbilical cord compression and others. Then, through obtaining the opportunity for timely intervention, obstetricians have believed that they could eliminate poor prognosis such as fetal and neonatal death, neonatal asphyxia, other neonatal morbidity and subsequent neurologic impairment including cerebral palsy.

This paper presents current consensus on the benefit and demerit of intrapartum electronic monitoring based on actual results of its clinical use for about 30 years, and what should be done for the future advancement.

\section{Effect of FHR Monitoring on Short- term Prognosis}

With the liberal use of electronic FHR monitoring, a number of studies were conducted on its actual benefit. $\mathrm{NIH}$ of united states reported a review of 11 studies including almost 40,000 monitored patients and nearly 100,000 historical controls and claimed the reduction in fetal death rate from $0.176 \%$ in control to $0.054 \%$ in monitored patients. ${ }^{2}$ Similar reduction in neonatal death rates was also reported. After these studies, in late 1970 s, other series of study were conducted focusing on the comparison of effects with another intensive fetal care method that is intermittent auscultation ${ }^{3 \wedge \ddot{y} 5}$. No differences in intrapartum fetal death rates were found in the population including both low and highrisk patients. In contrast, Vintzileos ${ }^{6}$ reported a significant reduction in perinatal deaths due to asphyxia in electrically monitored group in randomized, controlled trial. He claimed that the previous studies in which no different fetal death rates were found between electronically monitored and intermittent auscultation groups included only a few high-risk patients, so that the results must have represented data of low-risk patients.

Another important matter is that a nurse was assigned to each mother, that is one to one nurse to patient ratio in the intensive intermittent auscultation group and nurses auscultated the FHR at least every 15 minutes in the first stage of labor and every 5 minutes in the second stage. This type of patient care may be impractical and is impossible in usual clinical situations, so that electronic FHR monitoring is believed useful and rather convenient for improving fetal outcomes, particularly in high-risk cases.

As for neurological damage due to fetal asphyxia, MacDonald $^{7}$ suggested in 1985 that electronic monitoring may decrease the rate of seizures in the newborn.

In Showa University Hospital, FHR monitoring for lowrisk patients has been selectively performed at the time of admission with outset of labor, rupture of membrane, abrupt increment of labor pain, second stage of labor and other particular events. Table-'! shows rates of emergent cesarean section due to nonreassuring FHR pattern in low-risk patients. One out of 57 cases with cesarean delivery had severe neonatal asphyxia. This case was found to have velamentous cord insertion after delivery. At the beginning time of introducing electronic monitoring, fetal asphyxia during labor due to placental dysfunction was the main target to detect. But, recently, such cases are able to be diagnosed antenatally. We can identify depressed fetuses with use of nonstress test, biophysical profile score and so on before onset of labor. Therefore, nowadays, fetal asphyxia due to cord factor is most important to indentify during labor, which is still not easy to diagnose antenatally, then the cases with which are mostly deemed at low-risk. This implies the significance of selective application of FHR monitoring even in lowrisk patients.

Correspondence

Dr. Takashi Okai, MD, PhD

Prof. Department of Obstetrics \& Gynecology, Showa University School of Medicine

Email: okai.t@med.showa-u.ac.jp,Tel: +81-3-378486780 


\section{Effects of FHR Monitoring on Long- term Prognosis}

In 1996, Nelson and et $\mathrm{al}^{8}$ published their paper in New England Journal of Medicine. Among 155,000 children born in California, they found 95 children with cerebral palsy (CP). CP children were compared with randomly selected control children with respect to characteristics of FHR patterns noted in birth records. The results showed that patterns associated with increased risk of CP were multiple late decelerations (odds:3.9) and decreased variability (odds:2.7). However, predictive value of theses patterns for $\mathrm{CP}$ was $0.05 \%$ in low-risk cases, $0.25 \%$ in high-risk cases, and $0.19 \%$ in total, with false positive rate of $99.8 \%$. Huge numbers of cesarean sections were performed in total subjects, then with the assumption that the cesarean section was not beneficial when the cesarean section had been performed in the cases without $\mathrm{CP}$, nonbenefical interventions for prevention of one CP reached 2324 cases. With these results, the paper concluded that concerning prevention of CP, electronic FHR monitoring dose not appear to effective, as many cesarean sections would be performed without benefit and with the potential for harm.

Other researchers ${ }^{9,10}$ also reported no effect in preventing adverse long-term neurologic outcomes in their papers. The reason for little effects on prevention of CP is thought that perhaps only 3 to $20 \% \mathrm{CP}$ infants born at term are due to intrapartum asphyxia; in addition to that $\mathrm{CP}$ originally is relatively rare, so that it must be difficult to raise the efficiency. Furthermore, the mechanism of occurrence of $\mathrm{CP}$ has not been clarified yet and its relation with FHR pattern can be said almost completely unknown.

\section{Problems in Electronic FHR Monitoring at Present}

Today's consensus about problems in intrapartum FHR monitoring is summarized in Table-2. Electronic monitor is more convenient than intensive intermittent auscultation, with one to one nurse to patient care and every 15 minutes auscultation. However, patients who have a monitor adjacent to their bed are often bothered and stressed by difficulty in movement and sometimes

Table I. Rates of Emergent Cesarean Section in Low॰risk Cases at Showa University Hospital

\begin{tabular}{lc}
\hline year & c/s rate (no. of cases) \\
\hline 2000 & $1.66 \%(11 / 663)$ \\
2001 & $1.91 \%(13 / 680)$ \\
2002 & $2.22 \%(16 / 721)$ \\
2003 & $2.22 \%(17 / 764)$ \\
\hline total & $2.02 \%(57 / 2828$ \\
\hline
\end{tabular}

by beating sound. In contrast, nursing attention to the patient with respect to maternal comfort, emotional support, and "laying on of hands could have a significant impact on the patient's amenity. Therefore, electronic monitoring should be applied selectively and we have to improve the way of placing the monitor probe.

The second problem is a medico-legal and social issue. Electronic FHR monitoring is not perfect to prevent fetal adverse outcomes, particularly long-term neurologic impairment. There is still ambiguousness in interpretation of FHR patterns. Nevertheless, general people and even doctors who are not specialized for perinatology tend to be too confident of its effect. Thus, when the prognosis of the baby is adverse, they often deem the result obstetrician's fault as miss-judging or delay of obstetric intervention. This fact has already become a big social problem.

Increase in cesarean section rate is also a big problem, if most of them are not beneficial. One of the reasons for increase in cesarean section rate is that we have had criteria for obstetric intervention which are not completely correct or very adequate. Until recent year, it has been said that such four FHR patterns as late decelerations, severe variable decelerations, continuous bradycardia and loss of variability are ominous signs and prompt intervention has been required. Since several years ago, perinatal academic committees in various countries have been working to correct or modify these criteria for prompt obstetric intervention. NICHD (National Institute of Child Health and Human Development) concluded that the normal FHR tracing has normal baseline, normal variability, presence of accelerations and absence of decelerations, and in contrast, patterns predictive of fetal asphyxia are recurrent late decelerations, recurrent variable decelerations or substantial bradycardia with absent variability. Many fetuses have FHR patterns that are intermediate between these two extremes. Among them, those with patterns which conform to the past criteria for fetal asphyxia do not always indicate fetal hypoxia or acidosis. Then, American College of Obstetricians \& Gynecologists has proposed to name them "nonreassuring FHR patterns" in stead of "fetal distress".

Table 2. Problems in Electronic FHR Monitoring at Present

1) 'discontent with patients' amenity during labor

2) medico-legal and social issue

3) little effects on prevention of long-term neurologic impairment

4) increase in cesarean section rate 
Table 3. FHR Variability and $\mathrm{pH}$ of Umbilical Artery Blood in Cases with Decelerations Committee on Perinatology, Japan society of OB/GYN)

\begin{tabular}{lc}
\hline variability & $\begin{array}{c}\mathrm{pH} \text { of umbilical artery blood } \\
(\text { mean } \pm \text { SD) }\end{array}$ \\
moderate & $\mathrm{n}=10227.267 \pm 0.093$ \\
minimal $(\mathrm{n}=149)$ & $7.249 \pm 0.118$ \\
absent $(\mathrm{n}=5)$ & $7.078 \pm 0.274$ \\
\hline
\end{tabular}

In Japan, committee on perinatology, Japan Society of Obstetrics \& Gynecology analyzed the relation between FHR variability and umbilical arterial blood $\mathrm{pH}$ in the cases with FHR decelerations indicating nonreassuring patterns such as late, severe variable and prolonged decelerations. Mean $\mathrm{pH}$ was highest in the cases with moderate variability and those with absent variability showed significantly lower $\mathrm{pH}$. (Table-3)

When we look at the number of cases with low $\mathrm{pH}$, incidence of below 7.1 was $40 \%$ in cases with absent variability, $8 \%$ with minimal and $5 \%$ with moderate variability. And when calculated for 7.0 of $\mathrm{pH}$, they were $10 \%, 5.3 \%$ and $1.0 \%$, respectively. (Fig-1)

With these results, we can conclude that even in the cases with FHR patterns indicating fetal distress by the past criteria, the incidence of fetal acidosis is not so high, as long as FHR base-line variability is preserved. However, a few of those with moderate variability are acidemic, then their presumed condition and clinical management are still controversial, although it is significant to know positive predictive values of each pattern for fetal acidosis.

\section{For the Future Improvement of FHR Monitoring}

What we should do for enhancing the value of FHR monitoring toward the future is to improve its reliability and validity. For achieving this, we have to translate the following ideas into actions. 1, Interpretation and classification of FHR pattern should be minute and detailed. Detailed analysis includes quantification of decelerations, duration of abnormal patterns, progress of such patterns and combination of each parameter so on. 2, Relation between each pattern and fetal condition should be investigated with understanding of physiological aspects. 3, Predictive values of each subclassified pattern for fetal asphyxia should be determined. 4, Guidelines for management of nonreassuring FHR patterns, which is simple and easy for clinicians to conform, should be established.

Investigators working in this academic field should take the responsibility for future advancement of FHR monitoring leading to the better maternal and fetal care during labor.

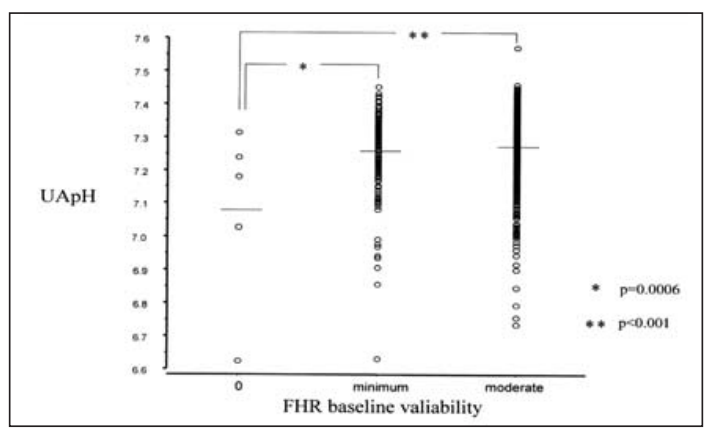

Figure I. Umbilical artery $\mathrm{pH}$ in cases with nonreassuring FHR pattern

\section{References}

1) Paul RH, Hon EH. Clinical fetal monitoring Vs Effect on perinatal outcome. Am J Obstet Gynecol 1974;118:529-33

2) National Institutes of Health. Antenatal diagnosis. Report of a consensus development conference. NIH publication \#79-1973. Bethesda, Maryland: NIH, 1979

3) Kelso IM, Parsons RJ, Lawrence GF, Arora SS, Edmonds DK, Cooke ID.

An assessment of continuous fetal heart rate monitoring in labor: A randomized trial. Am J Obstet Gynecol 1978; 131:526-532

4) Haverkamp AD, Orleans M, Langendoerfer S, McFee J, Murphy J, Thompson HE.

A controlled trial of the differential effects of intrapartum fetal monitoring.

Am J Obstet Gynecol 1979;134:399-412

5) Wood C, Renou P, Oats J, Farrell E, Beischer N, Anderson I. A controlled trial of fetal heart rate monitoring in a low-risk obstetric population. Am J Obstet Gynecol 1981; 141:527-534

6) Vintzileos AM, Antsaklis A, Varvarigos I, Papas C, Sofatzis I, Montgomery JT.

A randomized trial of intrapartum electronic fetal heart rate monitoring versus intermittent auscultation. Obstet Gynecol 1993; 81:899-907

7) Macdonald D, Grant A, Sheridan-Pereira M, Boylan P, Chalmers I. The Dublin randomized controlled trial of intrapartum fetal heart rate monitoring.

Am J Obstet Gynecol 1985; 152:524-539

8) Nelson KB, Dambrosia JM, Ting TY, Judith BS, Grether K. Uncertain value of electronic fetal monitoring in predicting cerebral palsy. $\mathrm{N}$ Engl J Med 1996; 334:613-618

9) Melone PJ, Ernest JM, O'Shea MD Jr, Klinepeter KL. Appropriateness of intrapartum fetal heart rate management and risk of cerebral palsy. Am J Obstet Gynecol 1991; 165:272-277

10) Colditz PB, Henderson-Smart DJ. Electoric fetal heart rate monitoring during labour: dose it prevent perinatal asphyxia and cerebral palsy? Med Austr 1990; 153:88-90 